\title{
Peptide receptor radionuclide therapy for neuroendocrine tumours: standardized and randomized, or personalized?
}

\author{
Michael S. Hofman • Rodney J. Hicks
}

Received: 16 October 2013 / Accepted: 18 October 2013 / Published online: 13 November 2013

(C) Springer-Verlag Berlin Heidelberg 2013

Neuroendocrine tumours are a heterogeneous group of tumours arising from a variety of specific chemical signalling cells in a range of organs. Superimposed on this intrinsic diversity of origin, they display a spectrum of biological behaviour from indolent well-differentiated tumours that may produce a variety of hormones, to aggressive poorlydifferentiated phenotypes that have rapid proliferation [1]. However, because of their collective rarity, they tend to get grouped into a single disease category for purposes of imaging and therapeutic trials. Peptide receptor radionuclide therapy (PRRT) is an evolving therapy but its utility is questioned by some because of the absence of prospective randomized controlled trials (RCTs). It sits amongst a growing number of therapeutic options, including pharmacological, hormonal [2] and liver-directed therapies, and surgical approaches. Pharmacological options include the mTOR inhibitor everolimus [3], the tyrosine kinase inhibitor sunitinib [4], and a variety of combination chemotherapy regimens, with promising results obtained with the combination of capecitabine and temozolomide in pancreatic NET in particular [5].

Amongst PRRT, there are also a myriad of options including a variety radioisotopes, different somatostatin analogues and combinations with radiosensitizing chemotherapy. Radiolabels include the Auger emitter ${ }^{111}$ In and the beta particle

M. S. Hofman · R. J. Hicks

Molecular Imaging, Centre for Cancer Imaging, Peter MacCallum

Cancer Centre, Melbourne, Australia

M. S. Hofman • R. J. Hicks

Department of Medicine, The University of Melbourne, Melbourne, Australia

M. S. Hofman $(\varangle) \cdot$ R. J. Hicks $(\square)$

Centre for Cancer Imaging, Peter MacCallum Cancer Centre,

St Andrews Place, Melbourne, Australia 3002

e-mail: michael.hofman@petermac.org

e-mail: rod.hicks@petermac.org emitters ${ }^{177} \mathrm{Lu}$ and ${ }^{90} \mathrm{Y}$; these have path-lengths of $0.02-10 \mu \mathrm{m}, 0.8-1.5 \mathrm{~mm}$ and $5-12 \mathrm{~mm}$, respectively. These can be administered alone or in combination, with emerging interest in alpha-emitters such as ${ }^{213} \mathrm{Bi}$. Somatostatin analogues include DOTA-TATE, DOTA-TOC and DOTANOC, with emerging interest in somatostatin antagonists such as pasireotide. Given this myriad of therapeutic strategies, how do we determine the optimal choice and sequencing of therapy in an individual patient? Should we rely on standardized approaches and RCTs to direct patient care, or is a personalized approach likely to be superior?

In the current issue of EJNMMI, Romer et al. compare the outcomes in 1,051 patients treated with ${ }^{90} \mathrm{Y}$-DOTA-TOC or ${ }^{177} \mathrm{Lu}$-DOTA-TOC [6]. Median survival was 35.9 and 45.5 months, respectively, with no statistically significant difference between the groups. Treatment was individualized with ${ }^{177} \mathrm{Lu}$ preferentially selected for patients with low tumour burden (up to three lesions), small lesions (diameter $<3 \mathrm{~cm}$ ) or poor kidney function (serum creatinine $>90 \mu \mathrm{mol} / \mathrm{l}$ ). This preselection of patients is quite appropriate based on theoretical considerations relating to the physical characteristics of these radionuclides and their differential potential for nephrotoxicity, but makes it difficult to draw robust conclusions between the two groups, as patients with more advanced disease and poorer prognosis were more likely to receive ${ }^{90} \mathrm{Y}$. Subgroup analysis adjusting for these confounders still suggested longer survival with ${ }^{177} \mathrm{Lu}$ than ${ }^{90} \mathrm{Y}$. However, we would contend that the fact that individuals with a larger disease burden did not fare worse than those with less advanced disease supports a tailored approach of individualizing the chosen radioisotope properties to the burden of disease and patient comorbidities rather than indicating superiority of one over the other.

The study adds to the growing literature supporting the use of PRRT. Despite a population that had been pretreated with surgery, chemotherapy and radiotherapy, the median survival 
was superior to survival following treatment with competing modalities. In this large cohort of Romer et al., median survivals after ${ }^{177} \mathrm{Lu}$ and ${ }^{90} \mathrm{Y}$-DOTA-TOC were 46 and 36 months, respectively. These results are consistent with those found in numerous other trials of PRRT [7-15]. In clinical practice, we frequently observe favourable responses with PRRT in patients with symptomatic, progressive disease resistant to all conventional therapy and often despite poor performance status. Indeed, such extraordinary responses constitute level 1c evidence by the Oxford Centre for EvidenceBased Medicine CEBM) 'Levels of Evidence' criteria [16]. This is met when patients died before the treatment became available, but now some survive on it. Questioning the efficacy of PRRT in this setting reminds us of the satirical call for a randomized control of parachutes for gravitational free-fall [17]. The effectiveness of PRRT should not be questioned but rather, should be further explored as to when it should be optimally applied in the sequence of available therapies.

Conducting RCTs in patients with neuroendocrine tumours, which have numerous subtypes of differing biological behaviours, is fraught with difficulty. One of the main challenges is defining an appropriate endpoint, as this depends on the tumour characteristics and the clinical indication for treatment. Progression-free survival is not a logical endpoint for someone without objective evidence of progression over a prolonged interval prior to therapy. Conversely, assessing symptoms in a patient with progressive disease who is asymptomatic is not sensible, except to assess toxicity. Consider a patient with severe carcinoid syndrome including debilitating diarrhoea refractory to conventional therapies who has complete resolution of symptoms following PRRT. Such a patient with high levels of hormone secretion typically has a welldifferentiated phenotype with an indolent growth pattern. The patient may not have a measurable response by scintigraphic or anatomic imaging criteria, and therefore shows no change in traditional measurements of image-defined "progression free survival". Further, the patient typically has a long life expectancy, rendering changes in overall survival difficult to determine. Consider by contrast a patient with more poorly differentiated disease, ENETS grade II/III, who presents with pain due to growth of metastatic disease. Following PRRT, symptoms improve drastically but there is significant enlargement of measurable disease on $\mathrm{CT}$, classified as progressive disease. FDG and DOTA-TATE PET/CT demonstrate a major response, characterizing the anatomic enlargement as due to cystic necrosis, not progression. In the context of a RCT with traditional 'hard' endpoints defined by anatomic RECIST criteria, both these patients would erroneously appear not to have benefited despite dramatic clinical benefit.

The selection of patients who might benefit from PRRT highlights the inherently personalized nature of this therapy. The delivery of and likely response to therapy can be predicted with high confidence by the use of imaging. The same compound as used for therapeutic targeting can be accurately imaged prior to and following treatment to both select the treatment and monitor its effects. This is a paradigm that has served nuclear medicine well from its earliest therapeutic application: the treatment of thyroid cancer with ${ }^{131} \mathrm{I}$. A patient with high uptake on somatostatin receptor (SSTR) SPECT or PET imaging, without spatially discordant FDGavid poorly differentiated disease that cannot be targeted [18], is highly likely to have a favourable response to therapy. Moreover, the intensity of uptake on pretherapy imaging, being a surrogate for lesional dosimetry with therapy, also correlates with outcome [12]. Contrast this theranostic approach with conventional chemotherapy, whereby the likelihood of response is not individualized but estimated by the average response of a patient cohort defined by a prior RCT. The mean of such a heterogeneous population may provide little insight into the likely response in an individual patient.

Our facility provides a highly personalized approach, adjusting administered activity, choice of therapeutic isotope, sequencing and frequency of PRRT to an individual's imaging phenotype, clinical course and pathological grade. We adjust administered dose based on the burden of disease defined on the basis of SSTR imaging, patient weight and renal function. With respect to actual dosimetry, we have found using quantitative SSTR PET/CT that a so-called "standardized" administered activity (which many erroneously call the "dose") may result in toxicity in patients with a small volume of disease, whilst significantly undertreating patients with a large volume of disease [19]. We select an isotope with an appropriate pathlength based on the pattern and bulk of disease on imaging, using ${ }^{111} \mathrm{In},{ }^{177} \mathrm{Lu}$ and ${ }^{90} \mathrm{Y}$, or a combination for small-volume, small-to-moderate and large lesions, respectively. We avoid ${ }^{90} \mathrm{Y}$ in patients with poor renal function, especially if they have reduced renal cortical width that brings more of the residual nephron mass within range of the beta particles emitted from the proximal convoluted tubules in the juxtamedullary region of the kidney. We also avoid ${ }^{90} \mathrm{Y}$ in patients with diffuse marrow involvement in whom crossfire to normal marrow increases the risk of haematological toxicity. We use a shorter time period of 6-8 weeks between cycles of PRRT in patients with higher Ki-67, compared to 8-12 weeks in patients with an indolent phenotype. We individualize radiosensitizing chemotherapy, using 5-fluorouracil [20], capecitabine, a combination of capecitabine and temozolomide or etoposide, depending on NET subtype. Such an approach is open to criticism by purists wedded to the current dogma that places the RCT as the highest level of evidence for medical interventions and will likely provide a challenge when negotiating current paths to regulatory approval and reimbursement. Nevertheless, we contend that it is strongly underpinned by scientific principles and a rigorous understanding of the physical and biological characteristics of the agents being used and an indepth evaluation of our patients. 
We concede that RCTs provide invaluable information when the balance between benefits and risks is uncertain. The lack of RCTs, however, must not be used to deny the strong evidence base and experience that has accumulated demonstrating remarkable responses to PRRT in patients who have failed other therapies. We congratulate Romer et al. on their efforts to further refine our knowledge about the potential benefits and risks of different PRRT regimens. Now that new systemic therapies are being developed that might have efficacy similar or superior to PRRT, RCTs are becoming relevant. In Australia, we are planning such a trial comparing PRRT alone to PRRT plus CAPTEM chemotherapy, and CAPTEM alone in patients with G2 pancreatic NET. However, we do not believe RCTs will be helpful in finetuning applications such as choice or administered dose of radioisotope where a personalized approach based on an individual patient's phenotype is clearly warranted.

Conflicts of interest None.

\section{References}

1. Hicks RJ. Use of molecular targeted agents for the diagnosis, staging and therapy of neuroendocrine malignancy. Cancer Imaging. 2010;10 Spec no A:S83-91. doi:10.1102/1470-7330.2010.9007.

2. Rinke A, Muller HH, Schade-Brittinger C, Klose KJ, Barth P, Wied $\mathrm{M}$, et al. Placebo-controlled, double-blind, prospective, randomized study on the effect of octreotide LAR in the control of tumor growth in patients with metastatic neuroendocrine midgut tumors: a report from the PROMID Study Group. J Clin Oncol. 2009;27:4656-63. doi:10.1200/JCO.2009.22.8510.

3. Yao JC, Shah MH, Ito T, Bohas CL, Wolin EM, Van Cutsem E, et al. Everolimus for advanced pancreatic neuroendocrine tumors. N Engl J Med. 2011;364:514-23. doi:10.1056/NEJMoa1009290.

4. Raymond E, Dahan L, Raoul JL, Bang YJ, Borbath I, LombardBohas C, et al. Sunitinib malate for the treatment of pancreatic neuroendocrine tumors. N Engl J Med. 2011;364:501-13. doi:10. 1056/NEJMoa1003825.

5. Strosberg JR, Fine RL, Choi J, Nasir A, Coppola D, Chen DT, et al. First-line chemotherapy with capecitabine and temozolomide in patients with metastatic pancreatic endocrine carcinomas. Cancer. 2011;117:268-75. doi:10.1002/cncr.25425.

6. Romer A, Seiler D, Marincek N, Brunner P, Koller MT, Ng QK, et al. Somatostatin-based radiopeptide therapy with [177Lu-DOTA]-TOC versus [90Y-DOTA]-TOC in neuroendocrine tumours. Eur J Nucl Med Mol Imaging. 2013. doi:10.1007/s00259-013-2559-8.

7. Brizzi MP, Berruti A, Ferrero A, Milanesi E, Volante M, Castiglione $\mathrm{F}$, et al. Continuous 5-fluorouracil infusion plus long acting octreotide in advanced well-differentiated neuroendocrine carcinomas. A phase II trial of the Piemonte oncology network. BMC Cancer. 2009;9:388. doi:10.1186/1471-2407-9-388.
8. Claringbold PG, Brayshaw PA, Price RA, Turner JH. Phase II study of radiopeptide $177 \mathrm{Lu}$-octreotate and capecitabine therapy of progressive disseminated neuroendocrine tumours. Eur J Nucl Med Mol Imaging. 2011;38:302-11. doi:10.1007/s00259-010-1631-x.

9. Delpassand ES, Samarghandi A, Mourtada JS, Zamanian S, Espenan GD, Sharif R, et al. Long-term survival, toxicity profile, and role of F$18 \mathrm{FDG} \mathrm{PET} / \mathrm{CT}$ scan in patients with progressive neuroendocrine tumors following peptide receptor radionuclide therapy with high activity In-111 pentetreotide. Theranostics. 2012;2:472-80. doi:10. 7150/thno.3739.

10. Imhof A, Brunner P, Marincek N, Briel M, Schindler C, Rasch H, et al. Response, survival, and long-term toxicity after therapy with the radiolabeled somatostatin analogue [90Y-DOTA]-TOC in metastasized neuroendocrine cancers. J Clin Oncol. 2011;29:2416-23. doi: 10.1200/JCO.2010.33.7873.

11. Krenning EP, Kwekkeboom DJ, Bakker WH, Breeman WA, Kooij PP, Oei HY, et al. Somatostatin receptor scintigraphy with [111InDTPA-D-Phe1]- and [123I-Tyr3]-octreotide: the Rotterdam experience with more than 1,000 patients. Eur J Nucl Med. 1993;20:71631.

12. Kwekkeboom DJ, Teunissen JJ, Bakker WH, Kooij PP, de Herder WW, Feelders RA, et al. Radiolabeled somatostatin analog [177LuDOTA0,Tyr3] octreotate in patients with endocrine gastroenteropancreatic tumors. J Clin Oncol. 2005;23:2754-62. doi: 10.1200/JCO.2005.08.066.

13. Cwikla JB, Sankowski A, Seklecka N, Buscombe JR, NasierowskaGuttmejer A, Jeziorski KG, et al. Efficacy of radionuclide treatment DOTATATE Y-90 in patients with progressive metastatic gastroenteropancreatic neuroendocrine carcinomas (GEP-NETs): a phase II study. Ann Oncol. 2010;21:787-94. doi:10.1093/annonc/ mdp372.

14. Horsch D, Ezziddin S, Haug A, Gratz KF, Dunkelmann S, Krause BJ, et al. Peptide receptor radionuclide therapy for neuroendocrine tumors in Germany: first results of a multi-institutional cancer registry. Recent Results Cancer Res. 2013;194:457-65. doi:10.1007/978-3642-27994-2 25.

15. Seregni E, Maccauro M, Coliva A, Castellani MR, Bajetta E, Aliberti $\mathrm{G}$, et al. Treatment with tandem [(90)Y] DOTA-TATE and [(177)Lu] DOTA-TATE of neuroendocrine tumors refractory to conventional therapy: preliminary results. Q J Nucl Med Mol Imaging. 2010;54: 84-91.

16. Oxford Centre for Evidence-based Medicine - Levels of Evidence (March 2009). http://www.cebm.net/?o=1025. 2009.

17. Smith GC, Pell JP. Parachute use to prevent death and major trauma related to gravitational challenge: systematic review of randomised controlled trials. BMJ. 2003;327:1459-61. doi:10.1136/bmj.327. 7429.1459.

18. Hofman MS, Hicks RJ. Changing paradigms with molecular imaging of neuroendocrine tumors. Discov Med. 2012;14:71-81.

19. Beauregard JM, Hofman MS, Kong G, Hicks RJ. The tumour sink effect on the biodistribution of 68Ga-DOTA-octreotate: implications for peptide receptor radionuclide therapy. Eur J Nucl Med Mol Imaging. 2012;39:50-6. doi:10.1007/s00259-011-1937-3.

20. Hubble D, Kong G, Michael M, Johnson V, Ramdave S, Hicks RJ. $177 \mathrm{Lu}$-octreotate, alone or with radiosensitising chemotherapy, is safe in neuroendocrine tumour patients previously treated with high-activity $111 \mathrm{In}$-octreotide. Eur J Nucl Med Mol Imaging. 2010;37:1869-75. doi:10.1007/s00259-010-1483-4. 